\title{
Improving Juvenile Justice Settings by Decreasing Coercion: One Lab's Perspectives from Behind the Fence
}

\author{
Odessa Luna' ${ }^{1} \cdot$ John T. Rapp ${ }^{2}(1) \cdot$ Kristen M. Brogan ${ }^{2}(1)$
}

Accepted: 5 January 2022 /Published online: 26 January 2022

(c) Association for Behavior Analysis International 2022

\begin{abstract}
In this article, we outline an emerging role for applied behavior analysis in juvenile justice by summarizing recent publications from our lab and discussing our procedures through the lens of coercion proposed by Goltz (2020). In particular, we focus on individual and group interventions that target a range of behaviors emitted by adolescents in a residential treatment facility. In general, individual interventions involve teaching adolescents to (1) respond appropriately to staff, (2) tolerate nonpreferred environmental conditions, and (3) control problematic sexual arousal. Likewise, group interventions involve low-effort manipulations that decrease disruptive behavior and increase appropriate behavior in settings with numerous adolescents. Thereafter, we describe behavioral interventions for staff working in juvenile justice. These staff-focused interventions aim to increase staff-initiated, positive interactions with students in order to change subsequent student behavior. In addition, we review our recent endeavors to assess and conceptualize other service providers' behavioral products (i.e., prescription practices) in a juvenile facility. Lastly, we discuss future directions of behavior-analytic intervention with juvenile-justice involved adolescents.
\end{abstract}

Keywords Applied behavior analysis · Crime · Juvenile justice · Prison · Rehabilitation · Skill acquisition

\footnotetext{
The authors thank Dr. Barry Burkhart for introducing our team to juvenile justice settings and the Alabama Department of Youth Services for their support. We are grateful and appreciative of Auburn University undergraduate and graduate students for their assistance, participation, and execution in these clinical endeavors.

Odessa Luna

odluna@stcloudstate.edu

1 St. Cloud State University, 720 4th Avenue South, Education Building A266, St. Cloud, MN 56301-4498, USA

2 Auburn City Schools, Auburn, AL, USA
} 
In 2017, the U.S. Office of Juvenile Justice and Delinquency Prevention reported that state and local officials committed or detained 43,580 youth in residential placements due to illegal behavior (Puzzanchera \& Hockenberry, 2019). It should be noted that males aged 15 years and older from historically disenfranchised communities were overrepresented. To address the unique needs of this population, U.S. juvenile justice facilities have seen a rise in screening for educational and mental health services (e.g., Hockenberry \& Sladkly, 2018). Although a number of groups have introduced positive behavioral supports models to juvenile detention settings (e.g., Boden et al., 2020; Jolivette et al., 2020; Jolivette \& Nelson, 2010; Sprague et al., 2020), behavior-analytic assessments and interventions have not been a part of those trends. Despite advancements in services, nearly half (44\%) of detention centers reported using mechanical restraints or isolation for more than 4 consecutive $\mathrm{hr}$ to control "unruly behavior" (Hockenberry \& Sladkly, 2018, p. 16).

The practice of imprisoning individuals for criminal behavior in the United States is articulated in the Congress of the National Prison Association's Declaration of Principles (1870), which states the "supreme aim of prison discipline is the reformation of criminals, not the infliction of vindictive suffering" (Article II, p. 1, 1870). In 2018 the U.S. Congress passed legislation, known as The First Step Act, to reform the criminal justice system for adults and youth in detention facilities and their families. As a part of this legislation, efforts were made to increase rehabilitation programming for offenders with the goal of decreasing recidivism. ${ }^{1}$ In particular, the First Step Act called for the evaluation of methods to improve academic and vocational education for offenders in prisons, jails, and juvenile facilities. However, as noted by the National Institute of Justice, criminal justice studies often (1) assume causation given correlation, (2) report anecdotal observations, or (3) employ methodology that lacks scientific rigor.

Researchers in applied behavior analysis (ABA) are well-equipped to study functional relations between behavioral change and intervention application (Kazdin, 2011). With our methodological practices, behavior analysts have made great strides in early behavioral intervention for children with autism (e.g., Perry et al., 2019) and assessment and treatment of problem behavior for individuals diagnosed with intellectual disabilities (Beavers et al., 2013; Chezan et al., 2018; Iwata et al., 1994). In light of these achievements, Apel and Diller (2017) lamented how broad applications of behavior analysis in criminal justice settings fizzled out in the 1980s. Nevertheless, Apel and Diller emphasized how behavior analysts could now make meaningful contributions to the criminal justice field by (1) working individually with adjudicated people and (2) applying group contingencies on a molar level. Likewise, Gendreau et al. (2014) conducted a meta-analysis of contingency management studies published between 1965 and 2004 and concluded contingency management interventions produced robust improvements in various resident behaviors in detention facilities.

\footnotetext{
1 As defined by the Federal Bureau of Prisons, recidivism is the extent in which a formerly detained individual engages in criminal acts that result in rearrests, reconviction, or return to a bureau of prisons within a 3-year period following release.
} 
In 1999, the Alabama Department of Youth Services (DYS) developed partnerships with Auburn University and the University of Alabama to provide therapeutic services to adolescents adjudicated for illegal sexual behavior (Alabama DYS, n.d.; Burkhart et al., 2009). At Auburn University, this partnership gave rise to the Accountability Based Sex Offense Prevention Program (ABSOPP; Brogan et al., 2018), which is directed by a licensed clinical psychologist who specializes in the treatment of youth who have been adjudicated of sex offenses. ${ }^{2}$ Although ABSOPP is affiliated with the Department of Psychological Sciences at Auburn University, it is housed within a juvenile justice facility. The facility contains a high school that offers a high school diploma, a general education development option, and trade classes. As a component of ABSOPP, our lab (i.e., the ABA team) began implementing and evaluating behavior-analytic interventions in 2016. The director of the Juvenile Delinquency Lab contacted the Program Director of the Auburn University ABA program (second author) to gain assistance and guidance when interacting with residents who were engaging in challenging behavior. The directors decided, as a trial run, they would have one ABA graduate student (third author) complete practicum hours with the ABSOPP team for 1 year (see Brogan et al., 2018 for more information about this preliminary collaboration). Over the past 5 years, the ABA team has developed various behavioral interventions to help justice-involved adolescents navigate the correctional environment, participate meaningfully in their required therapy, and leave the facility as soon as possible (within the constraints of their legally mandated therapy and sentence).

As central themes of our interventions, we have made efforts to minimize the use of aversive practices, justify our use of programmed reinforcers, and ensure transparency of our research and clinical endeavors with collaborating stakeholders. Through these practices, we embrace a scientist-practitioner model wherein we prioritize the use of positive reinforcement in settings in which staff, service providers, and administrators have historically relied on coercion (i.e., the use of negative reinforcement and punishment). Practitioners' control of antecedent and consequent events necessarily gives rise to concerns about undue control or influence. This issue is not uniquely applicable to the treatment of adolescents in residential treatment. For example, Leaf et al. (2021) outlined a number of issues stemming from the delivery of behavioral interventions for autistic individuals; some of these complaints emanate from perceptions of control and coercion. Likewise, Morris et al. (2021) highlighted the absence of a clear and consistent process for assenting participants with autism spectrum disorders and related developmental disabilities in behavior-analytic studies. By extension, standard practices for gaining clients' assent for clinical services by board certified behavior analysts are likely to be equally amorphous.

Discussions on the potential impact of coercion and restrictions to freedom are not new to the field of behavior analysis. For example, Goldiamond (1974) wrote extensively about societal concerns that stemmed from the legal community's fear of unwanted control and coercion from emerging techniques in behavior modification.

\footnotetext{
${ }_{2}^{2}$ The second author is currently the primary investigator for this service contract.
} 
In an attempt to reconcile this disconnect, Hayes and Maley (1977) highlighted critical differences between behavior-analytic and legal disciplines in the use of the term "coercion." From a legal perspective, they argued coercion may be determined, in part, by the salience or obviousness of influences and alternative options. From a behavior-analytic perspective, they argued a behaviorist should endeavor to "understand the conditions under which a term is used and analyze the function is serves" (Hayes \& Maley, 1977, p. 89). To that end, Hayes and Maley argued one social function of coercion was the controller's prevention of countercontrol by the controlee. We shall return to this issue later in this article. Others have elaborated on behavior-analytic perspectives of freedom, control, and coercion (e.g., Baum, 2017; Sidman, 1989, 1993). A thorough and exhaustive review of behavior-analytic conceptualizations of coercion and control is beyond the intention and scope of this article. Instead, we wanted to acknowledge the responsibilities inherent in the power imbalance that is created by the State when we provide assessment and treatment services for justice-involved youth.

In what follows, we frame our developing work in juvenile justice within recent behavior-analytic discussions of coercion. Because the recipients of our behavioral services are nonvoluntary residents of a juvenile facility, their choices are influenced by elements of power imbalance and coercion. In other words, it is unlikely residents would seek or otherwise assent to our services if they resided in a noncorrectional environment. This reality automatically places our behavior-analytic work within correctional settings at an extreme end of the coercion continuum (Goldiamond, 1976). However, we do not believe it precludes us or other behavior analysts from participating in the corrections process. To this end, Hayes and Maley (1977) opined the "behaviorist can be of aid in the development of behaviorally sound practices, where coercion must be used by the State" (p. 93).

Goltz (2020) recently extended the evolving behavior-analytic conceptualization of coercion. Our reading of the Goltz article provided both an opportunity for contextual reflection on the nature of our recent work, some of which involves our unequal power over residents' behavior and guidance for expanding behavior-analytic services within juvenile justice. Again, our intention is to illustrate how concepts outlined by Goltz (2020), as well as others (e.g., Goldiamond, 1976), influenced our treatment development, assessment of outcomes, or both, as opposed to providing equal coverage of all points raised by Goltz. In part, Goltz argued coercion should encompass instances in which those in power (1) fail to provide the resources to engage alterative behavior and (2) engage in libertarian ("soft") paternalism (i.e., choice architecture). An example of the former is when administrators in juvenile justice settings expect residents to behave in a polite manner toward staff, but fail to provide rules, reminders, and instructional sessions to ensure residents follow this expectation. An example of the latter is when a researcher fails to explain participation alternatives (i.e., opting out of the study) to a potential participant. Regardless of intent, the researcher (or practitioner) is nudging the participant to partake in their study (or treatment). The researcher assumes the participant would benefit from the study but fails to inform the participant of the range of choices available. Nevertheless, actions taken under the guise of soft paternalism ensure beneficial behaviors contact immediate reinforcers and in turn accumulate to contact distal reinforcers, 
avoid distal punishers, or both (see Lieberman, 2020, for a similar discussion regarding cardiovascular exercise). Given Goltz's proposed extensions to coercion and Apel and Diller's (2017) call for behavior-analytic action in criminal justice, it seems reasonable to analyze recent behavior-analytic studies within this framework and review how our lab's work with justice-involved adolescents drives our applied science.

The current article has three aims. First, we describe studies from our lab conducted in a juvenile justice setting to address residents, ${ }^{3}$ behavioral deficits and excesses. In general, individual interventions involve responding appropriately to staff, tolerating nonpreferred events, and controlling sexual arousal, whereas group interventions decrease disruptive student behavior and increase appropriate student behavior in settings with numerous adolescents. Thereafter, we describe behavioral interventions for juvenile facility staff. Throughout this article we identify the specific contextual need that gave rise to each intervention and infuse anecdotal reports 4 from residents and staff. Second, we discuss the extent to which our behavioral interventions and long-term outcomes are subject to Goltz's (2020) conceptualization of coercion. Third, we conclude by discussing implementation barriers we have encountered and potential avenues for future behavior-analytic research and practice in criminal justice settings. In the process of describing our experiences in a juvenile justice facility, we hope to inspire behavior-analytic research in other facilities.

\section{Behavioral Interventions for Individual Students}

As a part of ABSOPP, the ABA team receives referrals for behaviors ranging from passive noncompliance in classrooms during the day to toileting accidents in dormitories during the night. Teachers, clinical therapists, and dormitory supervisors most often refer students for (1) disrespectful behavior toward dormitory staff and teachers and (2) verbal or physical aggression directed toward facility staff, teachers, or other residents when preferred activities are either denied or unavailable. In addition, some students continue to emit problematic sexual behavior while in the juvenile facility. In the past, facility staff members responded to these disruptive behaviors with immediate, punitive consequences (e.g., reprimands, loss of privileges) and temporally distal consequences (e.g., damaged staff and teacher relationships, lower grades, and missed therapeutic services). These delayed consequences compounded over time and progressively worsened with repeated occurrences, which contributed to longer stays in the facility for residents. Due to the frequency in which we received referrals for disrespect and aggressiveness, the ABA team developed modules (i.e., teaching procedures) to address these behavioral excesses.

\footnotetext{
3 Because the ABA team provides behavior-analytic assessment and intervention in a therapeutic, educational context, we hereafter refer to these adolescents as either residents or students.

4 Anecdotal reports have not been held in high regard in applied behavior analysis in the past; our experience has reshaped how we view such information.
} 


\section{Self-Control Skills}

In behavior analysis, self-control is characterized as frequently engaging in behavior that results in larger-later reinforcers rather than behavior that results in smaller-sooner reinforcers (e.g., Rachlin, 2016). In juvenile justice settings, students are ubiquitously required to engage in appropriate behaviors under specific stimulus conditions without immediate social reinforcement to contact desirable consequences at a later time. In the following section, we describe studies that increased residents' adaptive behaviors (i.e., appropriate reactions to staff requests and delay tolerance to preferred events) and decreased residents' disruptive behaviors (e.g., excessive vocalizations, inappropriate sexual arousal).

\section{Appropriate Reactions}

The concepts of self-control (e.g., Rachlin, 2016), behavioral cusps, and interlocking contingencies informed our development of the appropriate reactions intervention. In essence, behavioral cusps are behaviors that expose a person to new environments, consequences, and opportunities to acquire new skills (e.g., Rosales-Ruiz \& Baer, 1997), whereas interlocking behavioral contingencies are situations in which a person's behavior, the behavior's consequences, or both function as an environmental variable in the contingency of another's behavior (Glenn, 1988, 2004). There were situations within juvenile facilities wherein staff members delivered unpleasant feedback or corrective statements to residents; this feedback, often evoked more severe problem behavior from residents, perhaps as a form of countercontrol (Delprato, 2002). Results for descriptive assessments suggested residents lacked the skill to receive feedback from staff members. To address this gap, Brogan et al. (2021) taught students to engage in an appropriate verbal response (e.g., "Yes/No, ma' am/ sir") when presented with directions or corrective statements from staff members. The intervention included all of the components of behavioral skills training (BST) before a 10-trial session during which ABA team members delivered feedback for correct and incorrect responses. Once a student acquired appropriate reactions in contrived sessions, they participated in additional sessions with staff members. Dormitory staff members reported residents became more pleasant after they completed our training. Those same residents reported staff in the dormitories offered them more choices (this component was not programmed by the ABA team). Thus, residents' engagement in an appropriate response, perhaps in part to acknowledging staff members' authority, may have given rise to decreased coercive practices.

\section{Momentary Arousal Suppression}

It is not surprising that some residents who are adjudicated for sexual offenses continue to engage in problematic sexual behavior within the juvenile facility (e.g., engaging in consensual and nonconsensual sexual acts with peers, masturbating while in class). In part, intervention for inappropriate sexual behavior within a juvenile facility involves inhibiting sexual behavior across most stimulus contexts, but permitting a narrow range of sexual behavior (in this context, 
masturbation only) in specific stimulus contexts (e.g., either while the resident is alone in their cell room and under the bed sheets or in the bathroom). To address inappropriate sexual behavior in the former contexts, Brogan et al. (2020) designed a procedure using methods similar to those described by Reyes et al. (2011) to teach three residents to decrease problematic sexual arousal. The intervention involved (1) providing education about sexual arousal, (2) conducting a baseline evaluation of self-reported sexual arousal while viewing nondeviant media, and (3) teaching students to engage in a counting exercise when they selfreported certain threshold of sexual arousal. Figure 1 shows the mean subjective unit of arousal across sessions during baseline and therapist instructions phases for Zaiden, Joseph, and Terry. During baseline, students' self-reported arousal remained above the criterion line (range: 3.8-9.0). Following the instructions from the ABA team members to count backwards from 100 or 50 if their selfreported sexual arousal was above 3 (Zaiden and Joseph) or above 5 (Terry), students' self-reported sexual arousal decreased while in the presence of ABA team members. In addition, two students engaged in suppression exercises even in the absence of the ABA team's instructions. It is possible the counting exercise elicited responses (e.g., controlled breathing) that competed with sexual arousal. On the other hand, counting may have momentarily decreased the reinforcing properties of media, thereby acting as an abolishing operation (e.g., Laraway et al., 2003) for sexual arousal. There has been debate surrounding the conceptualization and definition of motivating operations (e.g., Catania \& St Peter, 2019; Lechago, 2019; Miguel, 2019; Poling et al., 2019). The potential nuance of motivating operations conceptualization notwithstanding, a behavior-analytic account could contribute to important behavioral interventions for inappropriate sexual behavior.

Returning to the Brogan et al. (2020) study, it is important to note that the procedures' effectiveness was likely based on the student's motivation to decrease sexual arousal. In particular, students expressed interest in controlling their sexual arousal and participating in this assessment and intervention. Though students could opt out of the study without repercussions, none did so. To date, several students have participated in this intervention. In general, teaching students to count during periods of arousal could serve as a behavioral cusp. In particular, if an adolescent or young adult with this particular background (i.e., serving time in a residential facility) can effectively suppress arousal under conditions with preferred stimuli, they might be able to participate in other learning environments (e.g., vocational training or higher education). In turn, these experiences in educational environments might allow them to contact a range of novel reinforcers.

Despite promising outcomes of arousal suppression in Brogan et al. (2020), further research is needed to assess generalization of arousal suppression to nonsimulated contexts, which is complicated by federal and state laws forbidding direct measurement of sexual arousal for minors. In addition, it remains unknown if direct measurement of sexual arousal could result in negative emotional side effects, in particular given that many residents have experienced past sexual trauma. Nevertheless, researchers could track changes via permanent products (e.g., facility incident reports surrounding sexual behavior) across the residents' sentences. 
Fig. 1 Mean subjective unit of arousal for three ABSOPP residents. Note. Mean subjective unit of arousal (SUA), and the highest within-session SUA across baseline (BL) and therapist instructions phases for Zaiden (top panel), Joseph (middle panel), and Terry (bottom panel). The horizontal dashed line represents the criterion line in the therapist instructions phase for each participant. Reprinted with permission. Brogan, K. M., Rapp, J. T., Niedfeld, A. M., Thompson, K. R., \& Burkhart, B. R. (2020). Using arousal suppression exercises to decrease inappropriate sexual arousal in detained adolescent males: Three clinical demonstrations. Behavior Analysis in Practice, 13(2), 348-359. 10.1007/s40617020-00408-Z

\section{Summary and Analysis}

Goltz (2020) argues coercion should include instances in which individuals in power fail to provide resources for those not in power to engage in alternative behavior. By teaching residents to appropriately receive feedback and engage in another action when sexually aroused in group settings, the ABA team minimizes coercion by ensuring residents are able to meet the setting's expectations by engaging in appropriate, alternative behavior. The residents' skill acquisition enables them to navigate choice architecture systems that might not be transparent to them. In this way, we help residents side-step potential coercion they might encounter. However, the means by which we teach these skills, informing residents they can earn time with the ABA team, their favorite snacks, or extra time on the computer can be viewed as soft paternalism. We use these nudges to increase the momentary probability residents will participate in training sessions.

\section{Undesirable Stimulus Events}

Perhaps by design, juvenile justice environments contain a number of unpleasant events. Although two of the aforementioned modules address students' disrespectful behavior towards staffs' directives and corrective statements, some residents exhibited problem behavior when exposed to other events that are inherent of correctional settings. In particular, as a function of the highly monitored facility environments, students experience limited privacy and autonomy (e.g., being video recorded, spending all waking hours in close proximity to peers and staff), contrived rules due to isolated peer malfeasance (e.g., no talking during meals), and limited opportunities to talk to adults. Unfortunately, many of these undesirable stimulus events cannot currently be avoided. Moreover, failing to tolerate (defined below) these unavoidable events often results in further restriction of individual freedom and choice. After observing residents struggle with common situations in the correctional setting, we developed modules to teach students to tolerate these events.

\section{Tolerance Training}

Neil (2011) suggested compliant (or cooperative) behavior could be either "active" or "passive." In particular, Neil argued active compliance requires an individual to emit a specific response following an instructor's request, whereas passive 


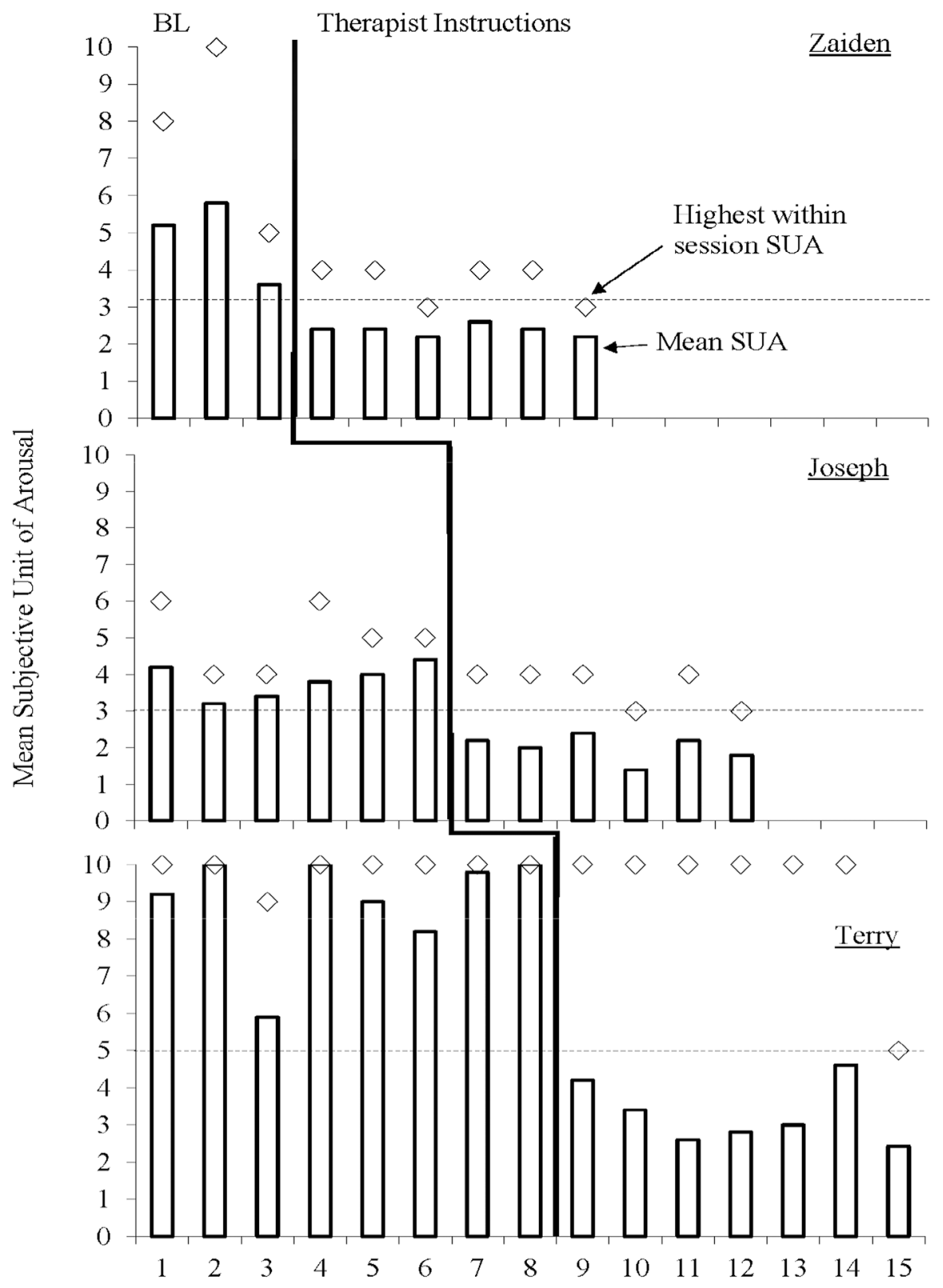

Sessions 
compliance does not. To this end, O'Rourke et al. (2021) adapted an intervention originally designed by Ghaemmaghami et al. (2016) to increase students' passive compliance ${ }^{5}$ or "tolerance skills" to aversive stimulus events. Facility staff members reported students engaged in disruptive behaviors (e.g., negotiating, property destruction, aggression) when they were either denied access to preferred activities or instructed to "deal with" nonpreferred situations (e.g., periods of low staff attention). In the presence of these nonpreferred stimulus events, each student's tolerance response involved (1) remaining in a designated seat or location and (2) not emitting additional behavior. We recognize the inherent issue with defining tolerance as the omission of any behavior (i.e., failing the dead person's test) deemed as disruptive. However, our team often uses this operational definition due to the demands and expectations of this juvenile justice setting. In particular, any active response from the resident could be perceived by staff as disrespectful and lead to unfavorable outcomes for the resident (e.g., additional peer comments, staff reprimands, or further escalation).

It is important to note that O'Rourke et al. (2021) modified procedures previously applied to individuals diagnosed with autism spectrum disorder (ASD; Cook et al., 2015; Rapp et al., 2005, 2017; Ricciardi et al., 2006; Schumacher \& Rapp, 2011). As the cornerstone of tolerance training, O'Rourke et al. taught students to request what they wanted when staff members presented a nonpreferred scenario and thereafter to tolerate situations in which their requests were denied (see Ghaemmaghami et al., 2016). In particular, the teaching contexts included student-specific unpleasant situations (e.g., removal of adult attention or preferred materials, presence of disorganized office materials ${ }^{6}$ ). A key procedural component of tolerance trainings was shaping the duration for which the resident tolerated the unpleasant event during planned sessions in a controlled setting. Using a trial-based method for systematically increasing the duration of the unpleasant event, all students reached the terminal criterion over 2 to 4 weeks. In addition, anecdotal reports from staff members indicated the students' tolerance behavior generalized to their dormitories. Indeed, learning when to sit still and wait is a necessary skill in correctional settings; however, this skill can also be applied outside the correctional setting.

\section{Signaling Events}

Although there are situations in which students are required to tolerate delays and denials without salient signals, signaling may increase treatment efficacy and be appropriate in some situations (see Betz et al., 2013, for one example). Niedfeld et al. (2020) implemented a stimulus control procedure in which team members signaled the nonavailability and availability of staff attention for excessive vocalizations.

\footnotetext{
5 As noted by Cook et al. (2015), "Passive compliance may involve teaching an individual to sit still, abstain from engaging in specific behavior, or otherwise tolerate an unpleasant event or stimulus" (p. 901).

${ }^{6}$ This study includes "resident zero," who was the impetus behind the development of an ABA unit. His inability to tolerate untidy environments was particularly problematic for other residents and staff members.
} 
The design of this stimulus control procedure was also informed by various studies with individuals diagnosed with ASD (e.g., Cook et al., 2014; Doughty et al., 2007; Lydon et al., 2017; Piazza et al., 1996; Rapp et al., 2009; Tiger et al., 2017).

Niedfeld et al. (2020) received referrals from dormitory staff regarding two students in the juvenile detention facility who engaged in excessive vocalizations. ${ }^{7}$ Although the vocalizations did not necessarily contain inappropriate content, the vocalization's frequency and duration prevented staff from completing work tasks. In an attempt to reduce work disruption, staff reprimanded the students for these vocalizations. Over time, these excessive vocalizations reduced the amount of positive interactions staff had with those particular students. Though most students in this juvenile justice facility emit complex verbal behavior, they often fail to identify and respond appropriately to common social cues that indicated staff unavailability (e.g., staff filling out paperwork). Thus, the ABA team designed a stimulus control procedure with salient stimuli for these two students. In particular, we used cards to signal times in which reinforcement was or was not available for vocalizing toward staff members.

After direct and indirect assessment results suggested students' vocalizations persisted due to contingent attention from staff members, the ABA team employed a multiple schedule of reinforcement to gain stimulus control over each resident's behavior. ABA therapists taught students that staff members' attention for vocalizing was available only when a green card (GC; or no card) was present, and attention was unavailable when a red card (RC) was present. Figure 2 shows results of the treatment for one of the students, Terry. ABA therapists systematically increased the amount of time the RC was present (terminal duration of a 30-min schedule) and decreased the amount of time the GC was present (terminal duration of $5 \mathrm{~min}$ ). As shown in Fig. 2, Terry (one representative student from the study) engaged in vocalizations at differentially at higher level during GC time periods than RC time periods, even when presented challenge conditions (i.e., specific contexts in which excessive vocalizations were most problematic for the student such as when others were talking). Taken together, this intervention (1) allowed staff to address other components of their job requirement and (2) helped residents effectively navigate their dormitory settings.

\section{Summary and Analysis}

The ABA team is teaching residents to engage in alternative behavior when encountering undesirable events. It should be noted that the ABA team minimizes coercion by providing resources for residents to engage in alternative behavior while in the facility; however, the team has yet to determine whether residents engage in this alternative behavior outside of the facility. In addition, for tolerance training and signaling events protocols, the ABA team clearly engages in soft paternalism because some contexts within correctional settings contain limited choices. We

\footnotetext{
7 This study included a third student who had been recently released from a different facility; however, that individual received intervention outside of the residential facility.
} 


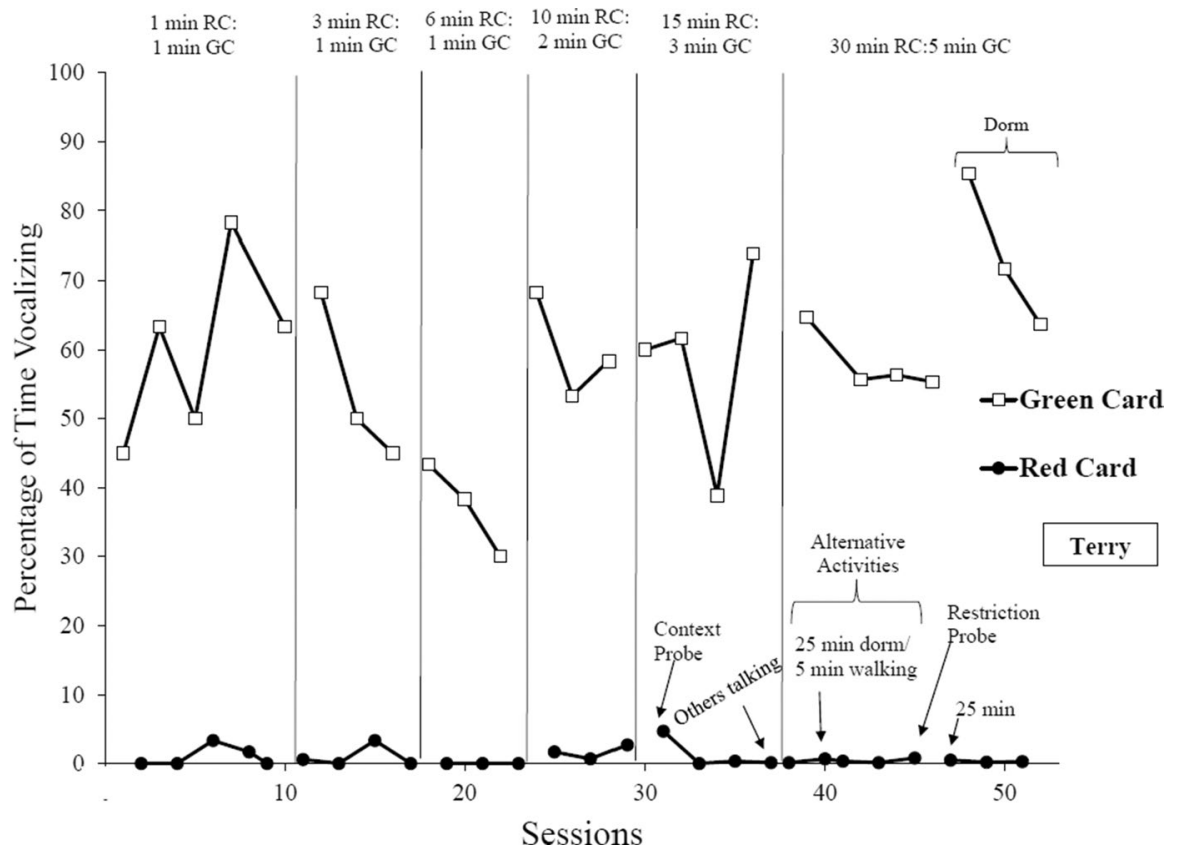

Fig. 2 Percentage of time vocalizing during red card and green card sessions. Note. $\mathrm{RC}=$ red card. GC = green card. Due to a weather event, RC session 40 does not have a corresponding GC session. Reprinted with permission. Niedfeld, A., Rapp, J. T., Coon, J. C., \& Cook, J. L. (2020). Using a multiple-schedule procedure to signal the availability of attention: Three demonstrations. Behavior Modification, 44(4), 496-517. 0145445519834640

presented a narrow range of choices: (1) "sit and wait" and gain access to attention or (2) fail to "sit and wait" and do not gain access to attention. As such, our actions appear to meet Goltz's (2020) definition of coercion by nudging residents to behave in a specified manner. Nevertheless, future programming could involve teaching the resident and staff members to engage in a range of different responses rather than the single choice we presented. For example, we could teach residents to engage in a request to terminate the unpleasant events or teach them to request access to a competing activity in addition to the waiting response. Likewise, we could teach staff to offer residents options to avoid unpleasant events. By teaching staff and residents a range of alternative behaviors, we might further reduce coercion in our programming.

In sum, the ABA team has decreased disrespectful and aggressive behavior in juvenile justice settings with numerous students using these modules. The ABA team continues to infuse the behavior-analytic literature for individualized assessment, treatment, and measurement into our clinical endeavors with residents. We have characterized the acquisition and maintenance of the adaptive behavior as behavioral cusps, which engender access to new privileges and activities and create or enhance relationships with peers, staff, teachers, and therapists. We also illustrated how the 
decrease of impulsive behavior allows students to avoid immediate punitive consequences that often result in longer durations at the facility and damage relationships with staff and peers. Instead, our students have often contacted the "larger-later reinforcers" by successfully completing vocational, academic, and therapy opportunities. Through our individual student protocols, we have attempted to alter the maladaptive interlocking contingency that occurs when our students engage in disruptive behavior. Despite this progress, the ABA team recognizes individual behavior change is often undervalued in correctional settings. There has been a recent legislative push (First Step Act, 2018) to reform adult prison settings and staff training. Aligning with current national priorities, we have evaluated procedures that intervene on the "behaving unit" of a group of individuals. In the next section, we describe our efforts to assess and treat groups of students in juvenile justice settings.

\section{Behavioral Interventions for Groups}

Although many students require an individualized approach to assessment and treatment, the ABA team have found groups of students often engage in disruptive behavior (e.g., excessive horseplay, inappropriate language, interrupting) that presented challenges in therapy sessions, classrooms, and dormitories. Rather than assess and treat multiple individuals' behavior in the group, the ABA team opted to design behavior-reduction interventions to meet the goals and needs of the target setting for groups of residents (Brogan et al., 2017; Chinnappan et al., 2020; McDougale et al., 2019). In addition, we have explored the extent to which group interventions influence staff behavior and exert residual effects on students' disruptive and appropriate behavior.

\section{Residents' Behavior}

The ABA team has created several group interventions designed to decrease disruptive behavior of residents while minimizing intervention components for staff members who are responsible for instructing and supervising the residents. To date, the ABA team has employed interventions in three broad settings.

\section{Group Therapy}

The first application of group-based ABA interventions occurred during two different therapy groups (see Brogan et al., 2017). The interdependent group contingency involved the delivery of five rules and an explanation of a contingency to the students in the group. The contingency involved the delivery of a small reward (i.e., student choice of snack and 10 min of free time) at the end of the group session if the group decreased their disruptive behavior below the levels of the previous session. When describing the contingency (a variation of a percentile schedule in which reinforcer delivery for current behavior is based on how behavior ranks with recent prior performance; Galbicka, 1994) to the students, team members deliberately provided students the vague instruction to "do better" than they did the previous session. 
Despite these vague instructions, students were able to successfully meet the contingency during $100 \%$ of opportunities. Not only did the intervention decrease disruptive behavior in a stepwise manner, but both students and therapists in the group reported high levels of satisfaction with the intervention.

\section{Classrooms}

Although the procedures in Brogan et al. (2017) decreased disruptive behavior for two groups of residents, we discovered it was not possible to provide rewards in all settings within the facility. As such, the ABA team designed an iteration of the original procedure wherein ABA team members provided rules, signaled instances of disruptive behavior with tallies on a marker board, but did not provide rewards for appropriate behavior (Chinnappan et al., 2020). This group contingency iteration decreased students' disruptive behavior and subsequently yielded high scores of acceptability from teachers. In a follow-up study involving two classrooms, Hamrick, Richling, Brogan, et al. (2021a) found residents' problem behavior was not affected by obtrusive observation (i.e., ABA interns informing students they were being observed). The team subsequently found clearly stated rules prior to each class meeting decreased problem behavior in both classrooms and increased appropriate behavior in one classroom. It is noteworthy that both studies found teachers provided attention, without specific training, contingent on students' engagement in appropriate behavior during the intervention phases. As before, teachers in both studies provided high ratings of approval for both the procedures and outcomes.

\section{Dormitory}

In addition to group therapy and classrooms, dormitories house multiple students who, when together, may display disruptive behavior. As such, the ABA team evaluated a group intervention to increase appropriate line walking when residents are required to walk to different areas on the facility campus (McDougale et al., 2019). As noted by McDougale et al. (2019):

Transitions are a potentially problematic time in residential settings because individuals from various dorms (some of whom administrative staff deliberately separated) cross paths within close proximity. Although transitions are typically less than $5 \mathrm{~min}$ in duration, relatively minor events (e.g., a verbal insult) could rapidly give rise to severe behavioral events (e.g., a physical altercation involving multiple residents). (pp. 624-625)

Similar to the step-wise intervention initially described by Brogan et al. (2017), team members in the McDougale et al. (2019, Study 2) provided rules, feedback, and a small edible when residents increased their appropriate line walking ${ }^{8}$ in

\footnotetext{
${ }^{8}$ Defined as "each student (a) walking approximately $1 \mathrm{~m}$ (i.e., arm's length) from the student in front of him, (b) not touching any other student, and (c) holding his hands behind their back, in pockets, or carrying approved objects" (McDougale et al., 2019, p. 625),
} 


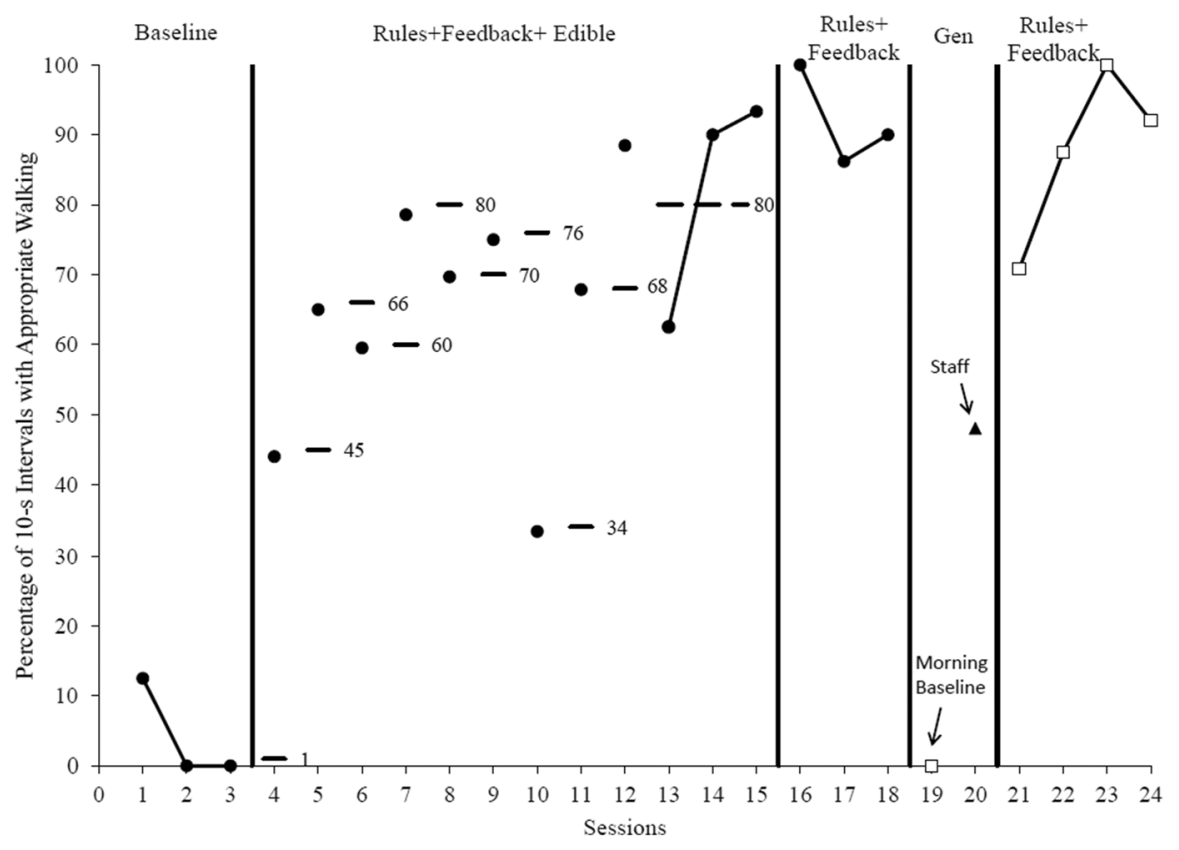

Fig. 3 Appropriate walking by residents during transitions. Note. Open squares denote novel transition sessions. The filled triangle denotes a session conducted by staff prior to formal training. Horizontal lines in the Rule + Feedback + Edible phase denote the response requirement for each session. Values next to each line denote the criterion. Gen = generalization probe sessions. Reprinted with permission. McDougale, C. B., Coon, J. C., Richling, S. M., O’Rourke, S., Rapp, J. T., Thompson, K. R., \& Burkhart, B. R. (2019). Group procedures for decreasing problem behavior displayed by detained adolescents. Behavior Modification, 43(5), 615-638. 10.1177/0145445518781314

comparison to the previous session. After several intervention sessions, residents displayed appropriate line walking during most excursions to specific locations on the campus without the use of edibles. As shown in Fig. 3, residents engaged in near zero levels of appropriate walking during baseline. During the first intervention phase residents' appropriate walking increased to the researcher-designated criteria of $75 \%$ of the opportunities. Ultimately, the team used the rules and feedback during these transitions and residents engaged in higher levels of appropriate walking (which corresponded to lower levels of inappropriate vocalizing, data not shown). Following the study, the ABA team also taught staff to implement this intervention. This intervention increased desirable behavior from multiple residents. Overall, the ABA team has implemented a variety of groupbased interventions that decreased disruptive behavior and increased desirable, safe behavior. 


\section{Summary and Analysis}

In Chinnappan et al. (2020) and Hamrick, Richling, Brogan, et al. (2021a), the ABA team did not provide the extra incentives (e.g., choice architecture) that would meet Goltz's (2020) definition of coercion. Instead, team members arranged antecedent stimuli to occasion alternative behavior that, in the presence of a teacher, was likely to contact attention. An alternative explanation is that the positive outcomes of this group contingency in McDougale et al. (2019) with the promise of rewards provided the necessary push (or nudge) for residents to engage in appropriate behavior. By structuring choices in this manner, the ABA team actions squarely fall within Goltz's (2020) definition of coercion. Nevertheless, all ABSOPP residents are legally required to complete ABSOPP therapy. Thus, our soft-paternalistic practices promoted timely completion of the required rehabilitation and the eventual release from the juvenile facility.

\section{Staff Members' Behavior}

Dormitory staff members spend more time with the residents than any other professional (e.g., teachers, behavior analysts, therapists, clinical psychologists) in correctional settings. As such, dormitory staff members might be the ideal service providers to create encouraging and affirming environments that promote residents' rehabilitation. In fact, positive staff-detainee interaction is negatively related to an offender's level of anxiety and feelings of helplessness in detention or rehabilitation settings (Biggam \& Power, 1997). Furthermore, positive social interactions between adjudicated individuals and staff members predict successful adaptation to rehabilitation environments (Liebling, 2004). In addition, group-based interventions allow staff members to work together toward a mutually beneficial outcome and reduce the effort associated with implementation (McDougale et al., 2019). Given these benefits, group behavior-analytic interventions may be an ideal fit for correctional staff members.

Our team members have experience training and interacting with change agents who work with children diagnosed with disabilities in school settings (Luna et al., 2018, 2019). It is worth noting that there are differences between juvenile justice and educational staff. In particular, staff for children diagnosed with ASD and related disabilities are typically motivated to alter their behavior to evoke appropriate child behavior. By contrast, correctional staff members, in our experience, are often disinclined, as indicated in their verbal behavior directed to our team, to change their behavior as a first step toward facilitating residents' behavior change. This disconnect became obvious as the ABA team extended services to general admission population (GAP) residents and staff (i.e., employees who work with adjudicated adolescents with histories of other criminal behavior). Although all facility staff members were at least 21 years of age and had minimum of a high school diploma or high school degree equivalency, staff members in ABSOPP dormitories were more likely to have longer tenure at the facility and received additional training from a highly 


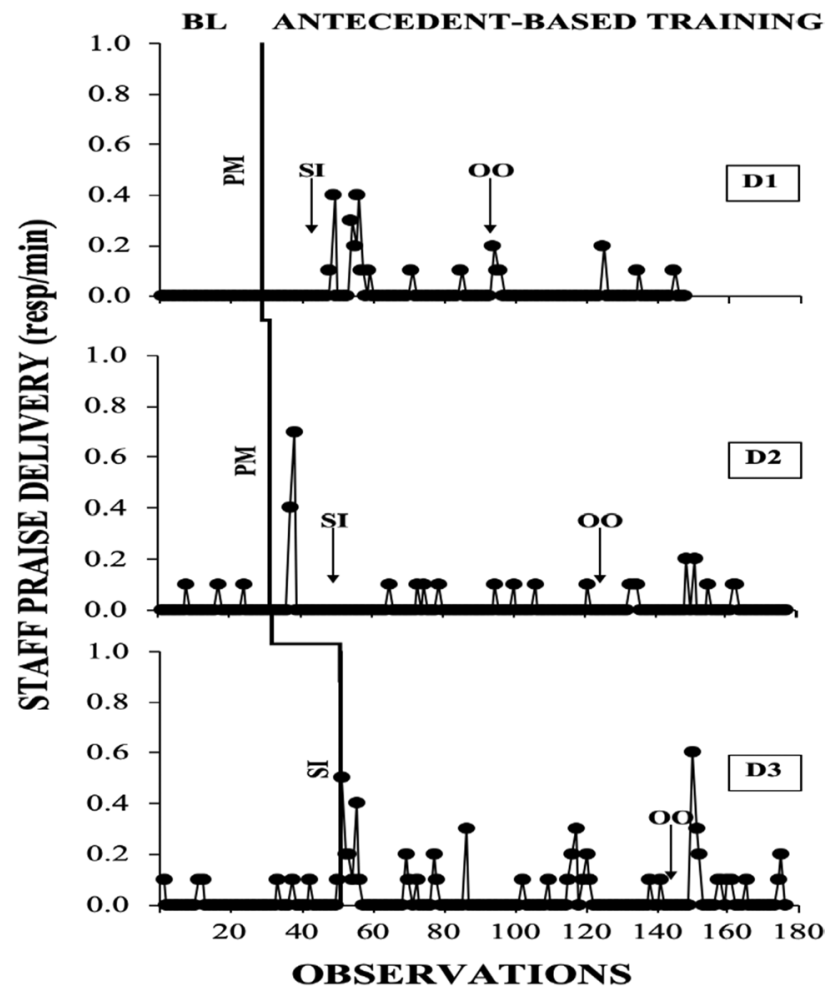

Fig. 4 Praise delivery across three dorms. Note. Arrows and text abbreviations indicate when and what the trainings occurred during Study $2(\mathrm{PM}=$ positive monitoring, $\mathrm{SI}=$ strategic interaction, and $\mathrm{OO}=$ offering options). Reprinted with permission. Luna, O., \& Rapp, J. T. (2020). Increasing praise delivery within dorms of a juvenile justice facility. Behavior Modification. Advance online publication. $10.1177 \% 2 \mathrm{~F} 0145445520982976$

experienced clinical director regarding resident interactions (see Luna \& Rapp, 2020). In short, as a likely outcome of their limited experience and training, GAP staff members were more likely to use coercive practices to control GAP residents' behavior. Likewise, they rarely provided constructive support (e.g., praise statements) to the GAP residents.

As a first step toward curbing coercive practices in GAP dormitories, we evaluated the effects of a progressive series of trainings to increase staff members' praise delivery across three residential settings for adolescents adjudicated for criminal offenses (Luna \& Rapp, 2020). Unlike traditional single-case experimental designs (Smith, 2012), ABA team members intervened on dormitory staff members as a group. In particular, each staff member's praise statements, reprimands, and instructions contributed to the total occurrence of behaviors within an observation. In particular, we recorded praise delivery when a staff vocalized an encouraging or affirming statement about resident behavior. We also scored resident disruptive behavior as a group. Prior to training, staff members from each 
of the dormitories engaged in low levels of praise delivery and resident engaged in variable levels of disruptive behavior. We conducted group trainings that instructed staff members to deliver positive social interactions with the residents by modeling respectful behaviors (e.g., using manners, avoiding cursing, using appropriate social gestures), frequently checking in with residents (e.g., using a vibrating timer to prompt check-ins), and offering leisure items during recreation periods.

As shown in Fig. 4, Luna and Rapp (2020) found the group-delivered staff training yielded only modest increases in praise delivery for two of the three dormitories, which corresponded to some differences in residents' appropriate behaviors following staff training for one dormitory. In baseline across the three dorms, staff member praise occurred at low levels, and the three trainings (positive monitoring, strategic interaction, and offering options) had only a marginal impact in influencing staff praise. Despite this less than positive finding, ABA staff aim to encourage, through evidence-based training, staff members to view themselves as an agent of change for the residents' lives.

Juvenile justice workers often enter and stay in correctional settings due to an aspiration to help rehabilitate adolescents (e.g., Howe et al., 2007). The transient effects of the training were likely due to competing cultural contingencies for staff to use coercive practices and regular staff turnover in this setting, among other issues. To address these barriers, further training evaluations could target instructing staff with longer histories at the facility. In this approach, the ABA team could provide training to more tenured staff members in a group, instruct them to model therapeutic practices (e.g., delivering social interaction to residents), and teach them to provide positive feedback to their colleagues engaging these practices. Researchers could subsequently determine whether this training is sustainable and effective in creating long-lasting staff and resident behavior change.

In a related study, Luna et al. (2021) evaluated residents' and staff members' time allocation to various leisure activities in four dormitories. As part of their analyses, Luna et al. (1) tracked whether residents and staff simultaneously engaged in activities and (2) categorized activities as providing opportunities for skill building, communication development, or both. Skill building activities contained materials staff could use to support residents in managing their daily lives inside or outside of the facility such as writing, reading, and completing academic tasks. Communicative activities included activities that facilitated reciprocal social exchanges between staff members and residents (e.g., board games). In the three GAP dorms, Luna et al. found residents allocated their time to activities that did not contain skill building or communicative activities. According to Goltz (2020), administration's failure to provide structural antecedents to evoked appropriate skill-building activity within the facility could be viewed as coercion toward staff members and residents. Based on these findings, Luna et al. suggested (1) dorm supervisors and other service providers should incentivize residents to participate in skill building activities and (2) facility administration prioritize training their staff to instruct residents to engage in these activities during leisure periods. To this end, facility administration has engaged in multiple corrective actions such as forming additional training groups for residents and allowing our team to conduct additional 
research on such activities within the facility. We can report that staff members appear enthusiastic about the ABA team's solutions.

\section{Summary and Analysis}

Goltz's (2020) definition of coercion can also be applied to the behavior of juvenile justice administrators and staff members. That is, if the administration fails to train staff to use low-coercion procedures to effectively manage residents' disruptive behavior, the staff might resort to using only methods with which they are familiar or that are supported by the environment (e.g., isolation and restraint). By contrast, specific procedures might be more likely to enhance staff members' relationships with residents, thereby creating cusps for staff. Nevertheless, due to the high cost of training, educational requirements, and constant turnover (Lambert, 2001; Lambert \& Hogan, 2009; Minor et al., 2011), correctional administrations might not train staff to use empirically supported procedures. Therefore, the ABA team has sought to use group-based interventions when training dormitory staff members.

\section{Behavior of Other Service Providers}

In addition to assessing the behavior of staff members, we received permission from facility administrators to evaluate their consulting psychiatrists' behavioral products. Consistent with other populations in state custody, many residents receive psychotropic medication during their stay in the juvenile facility. Moreover, psychotropic medication can exert overarching effects that impact the other services. Anderson et al. (2021) sampled residents' medication files from a 7-year period to determine the percentage of residents who received psychotropic medication, the type of psychotropic most frequently prescribed for residents, and the extent to which residents experienced a systematic decrease in the number of psychotropic medications prior to their discharge from the juvenile facility. Based on a sample of 135 former residents, Anderson et al. found (1) $42.2 \%$ received one or more psychotropic medications during their stay; (2) the most frequently prescribed psychotropic medications were stimulants, antidepressants, and antipsychotics; and (3) 38.6\% of residents who received psychotropic medication experienced discontinuation of all psychotropic medication prior to their discharge. In addition, Anderson et al. found residents who received antipsychotic medication were least likely to experience discontinuation of any medication. Figure 5 shows the results for five residents (3.7\% of all residents in the facility and $8.8 \%$ of residents prescribed psychotropic medication) who were prescribed stimulants and antipsychotic medication concurrently. It should be noted that these residents were prescribed other psychotropic medication and none of these residents received stimulants in isolation before physicians prescribed other medication.

\section{Summary and Analysis}

This juvenile justice facility's prescribing and deprescribing practices behavior align with Goltz's (2020) conceptualization of coercion. In particular, the site does not 


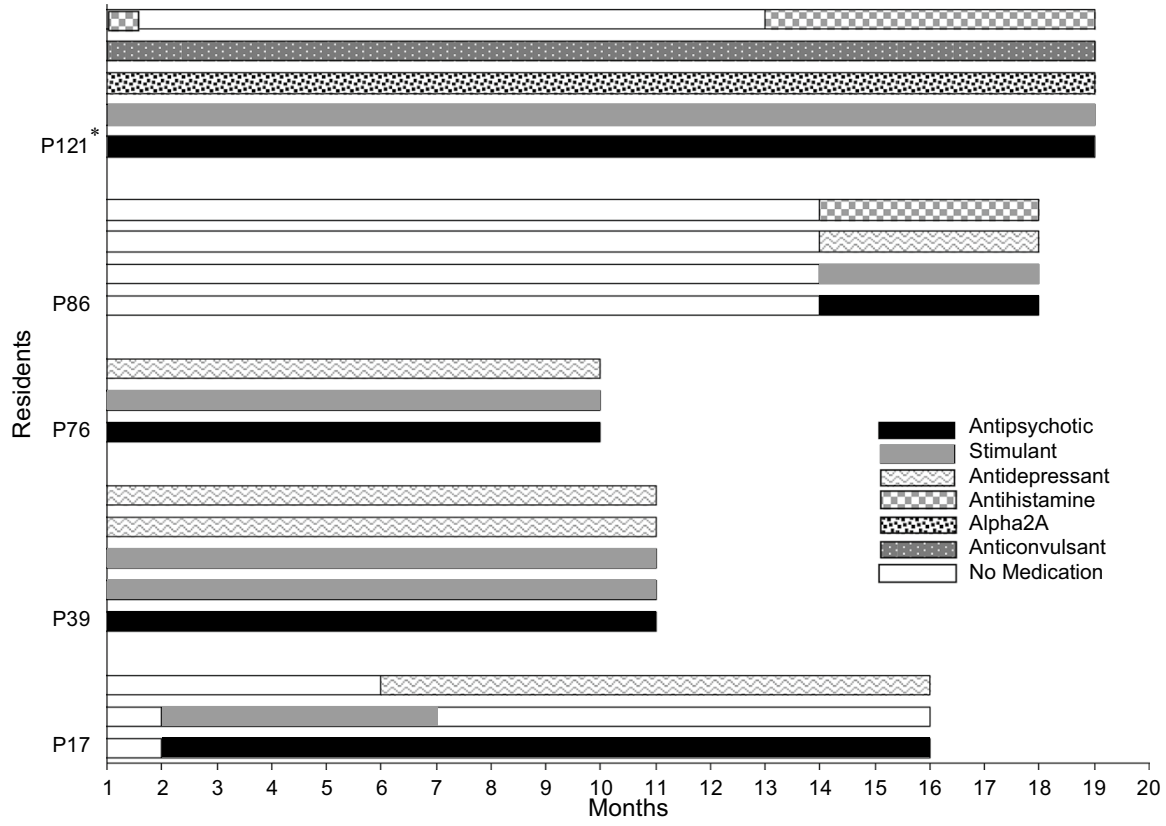

Fig. 5 Length of stay and medication for residents prescribed a stimulant and an antipsychotic. Note. Month 1 denotes the first month of residence. The end of the row denotes the month of discharge from the facility. Each row for the resident represents a different class of medication. Colors and patterns denote the different classes prescribed as outlined in the legend. The solid white portion of the medication row represents time served without receiving medication. The asterisk denotes resident had medications at intake. Reprinted with permission. Anderson, A., Rapp, J. T., \& Kierce, E. (2021). Psychotropic medication prescribing in a juvenile justice facility: Evidence of a limited discontinuation process. Advance online publication. Residential Treatment for Children \& Youth. 10.1080/0886571X.2021.1887789

provide educational opportunities for residents to learn about psychotropic medications effects (therapeutic and side effects) on private and public behavior and advocate for oneself during physician appointments. As a result, the site fails to provide necessary resources and interventions to residents to engage in alternative behavior during these physician interactions (e.g., asking questions regarding the purpose of medications and potential outcomes vs. passively complying). Furthermore, it is likely the physician is nudging or engaging strategic choice architecture occurring during appointments. That is, it is unclear if the physician or other service providers outline alternative supports to residents rather than psychotropic medication (i.e., behavioral intervention). It must be assumed that a physician prescribing is acting under soft paternalism for the benefit of the resident, providing medication support to assist with decreasing residents' disruptive behavior and increasing appropriate, to ensure the resident meet the behavioral and academic criteria to be released into their community.

Therefore, results from the Anderson et al. (2021) study have given rise to two avenues for further inquiry that are driven by facility administrators. First, facility 
administration expressed interest in treating conditions such as sleep disruption (one reason antidepressants were frequently prescribed) with behavioral interventions instead of psychotropic medication. Second, administrators expressed interest in developing a formal system of evaluating the effects of psychotropic medication, in particular those prescribed for aggressive behavior, for each resident. In part, this formal system will require the ABA team to (1) train facility staff members to reliably track target behavior and potential side effects and (2) develop a system for delivering consolidated information about each resident's behavior to the consulting psychiatrist in a manner that will facilitate rational prescribing. Finally, by conducting this study, the ABA team initiated a new interlocking behavioral contingency in the culture surrounding the use of psychotropic medication in this facility. That is, conducting the study gave rise to projects for enhancing and developing behavioral services for ABSOPP and GAP populations. Moreover, increasing service options likely decreases coercion for residents.

\section{Life after Residential Treatment}

Although studies have shown providing postincarceration or reentry programs (e.g., employment services, drug counseling) for adults decreases recidivism, recent problems such as limited funding and increased resident populations have decreased access to such programs (Apel \& Diller, 2017). Adolescents in residential treatment lose one or more formative years to detention that should have been devoted to developing preliminary employment and social skills. To address this deficit, again we have borrowed procedures from other areas of ABA. The goal of these protocols was to instill skills (behavioral cusps) that increased residents' future contact with appropriate education and employment and simultaneously decreased their contact with justice systems.

Noting that the interview process was the first link in a chain of behavioral events culminating in active employment, Edgemon et al. (2020) used behavioral skills training (BST), alone or in combination with modified prompting procedures, with seven residents to increase appropriate responses to interview questions, appropriate questions, correct posture, and smiling, and decrease fidgeting. Although state laws prevented Edgemon et al. from tracking adolescents after they are released, anecdotal information provided through family members indicated that at least two students used their skills for employment, education, or both.

A legal issue, broadly recognized as sex offender registration and notification (SORN) laws (Lytle, 2019), that looms over adolescents adjudicated for sex offences as they approach their release date is the requirement to report their offender status where they establish a residence. One way to evaluate the impact of this requirement on residents of juvenile facilities is to conduct simulations with nondetained individuals. In a study with nearly 500 college students on a hypothetical delay discounting task, Falligant and Pence (2019) found, among other outcomes, that when students' hypothetical charges involved sex-offender registration and notification compared to prison time, students were more likely to accept additional time toward their sentences in exchange for delaying the onset of the consequences. This 
finding suggests young adults perceive the process of registering as a sex offender as an aversive event. Although the generality of this finding to adolescent males with actual offenses is unclear, residents in the juvenile facility often express concerns (and anxiety) regarding the limitations that may be imposed on them following release.

Behavior analysts cannot undo choices that were previously made by residents; however, we can develop interventions to strengthen residents' repertoires to reduce future contact with justice systems. To this end, correct responding to questions about the state's sex laws is a requirement of the ABSOPP curriculum. Although some residents are able to produce correct responses to questions about state sex laws after completing a short, group course, many fail to achieve the minimum standard. As a result, these residents are prevented from advancing to the next level of the ABSOPP curriculum. In a response to this problem, Hamrick, Richling, Davis, et al. (2021b) used a fluency procedure to teach residents to respond correctly to questions regarding the state's sex laws. It is important to note Hamrick, Richling, Davis et al. employed mastery criteria that predict long-term maintenance when evaluating students' performance (Fuller \& Fienup, 2018; Richling et al., 2019). Although emitting correct responses to questions about state sex laws does not ensure that residents will emit "correct responses" when confronted with an actual event for which sexual stimulation is a high probability outcome and additional contact with justice systems is a lower probability outcome, our goal, which is consistent with soft paternalism (Goltz, 2020; Lieberman, 2020), is to bring residents' current behavior under control of rules whenever possible. In the absence of rule-governed behavior, we can assume students' behavior will gravitate toward immediately available reinforcers. Further research on the effects of these programs is needed.

\section{Avoiding Coercion}

Throughout this article, we have periodically infused concerns about coercion (Goltz, 2020) within the correctional culture. In keeping with these concerns, the ABA team balances the practice of providing incentives to the residents to participate in therapy sessions with the inherent need of residents to make individual choices. We provide incentives (i.e., preferred stimulus events) to improve the residents' therapy experience and produce changes in residents' repertoires while at the facility and, it is hoped, following their release. We question if we have assumed a paternalistic position such that we (our team) (1) know what is in each student's best interest and (2) should take all conceivable steps to influence students to make choices that comport with perception of their best individual interests. Nevertheless, we frequently encountered conflicting philosophical positions from other corrections service providers. One of which is the residents' right is to refuse to participate in therapy.

Our sessions with a resident typically involved (1) $5 \mathrm{~min}$ of noncontingent social interaction with ABA team members upon arrival; (2) 45 min of one or more specific training protocols focused on skills deficits, behavior excesses, or both; and (3) 5-10 min of noncontingent social interaction with graduate students and a snack 
of the resident's choosing. In short, actively choosing to attend, but not necessarily participate in, ABA sessions increased access to a number of potentially reinforcing events. We adopted this structure to develop rapport with residents to whom we deliver behavioral services (the second author had previously employed this practice in a school-based project). Could ABA team members' efforts to establish rapport with residents be construed as coercion? In particular, should we thin the schedule of preferred events during training assessment to avoid coercion stemming from the contrast in reinforcement density inside and outside of our training sessions? As an alternative, are there measures we could implement that could make other aspects of the correctional environment as enriching as our training sessions? The questions and the respective answers continue to evolve.

What we (behavior analysts) can and should do to address residents' behavior in correctional settings may be misinterpreted by other stakeholders. In general, we argue our team is attempting to alter the regular, correctional environment to include more reinforcing and fewer aversive events with our programming. By altering the correctional setting in this manner, using soft paternalistic practices (strategies often used in many societal structures), we recognize these behaviors are mildly coercive. We argue this low-level of coercion in the short term might yield positive benefits for the residents in the long term. If we were to prioritize eliminating all coercion to the extreme, this would reduce or eliminate our training options for residents. As behavior analysts, we are in a discipline of action. As more groups join this challenge, we believe the collective outcomes will give rise to a balanced understanding of how and what behavioral services should be available in juvenile justice settings.

\section{Barriers to Effective Action}

As a point of clarification, we believe it is pertinent to outline the various constraints we and others have experienced while conducting clinically directed research in residential juvenile justice facilities. To this end, we experienced (1) various forms of resistance from the "old guard," (2) unique constraints from the institutional review board (IRB) s pertaining to procedures and sharing of outcomes (see also Applebaum, 2008, and Lane et al., 2012, for broader discussions), and (3) problems disseminating our clinical studies. We briefly touch upon these issues below and informally describe our efforts to navigate each barrier.

In regard to the "old guard" concept, we use frequent staff interviews and outcomes of behavioral interventions as feedback for the development and refinement of subsequent interventions. Having made these efforts, one of the most common staff comments is "y'all seem to forget why these boys are here," which seems to suggest they view their role as guarding the residents as opposed to facilitating their behavior change. In addition, there is a tendency for some senior dormitory supervisors to view residents' behavioral issues, regardless of severity, as safety issues. This interpretation allows those individuals to circumvent behavior programming, and instead implement highly punitive measures (typically placing the offending resident in seclusion for an extended period of time). Of course, this action requires less effort, and presumably involves removing highly aversive stimulation (i.e., a resident 
emitting problem behavior). To address these different philosophical backgrounds and perspectives of staff members and administrators in juvenile corrections, we have learned to couch our procedures within the stated needs of the facility.

It is important to note that all participants in research are protect by IRB guidelines. Individuals in correctional settings are provided additional layers of protection by IRBs to minimize coercion in the participant enlistment process. To this end, IRBs permit three types of research in correctional settings. First, research can focus on the causes, effects, and processes of incarceration and criminal behavior. Second, research can consist of situations that affect particular prisoners as a class (e.g., prisoners who are also parents). Third, research can focus on practices that strive to improve the detainee's life. Our team's research has fallen almost exclusively into the third category. In fact, for a year, the second and third authors were the sole providers of behavior-analytic services for the most challenging residents at the facility. As previously noted, our research arises from the needs of the residents, staff, and the site. We did not enter the correctional facility with a concrete list of research questions developed a priori. Instead, we embraced the scientist-practitioner model with our work at the juvenile justice facility as our partner, where we prioritize the residents' needs to ensure we produce meaningful change in their lives. We believe this is the path behavior analysts who conduct research and provide clinical services in juvenile justice settings should follow. Every year, administrators within this facility actively choose to partner with the ABA program at Auburn University. Their active responding indicates their acknowledgement of the team's contributions and, perhaps, their commitment to decreasing coercive practices with the juvenile facility.

It should be noted that our team is restricted by what kind of detail our articles can include about the students (to protect confidentiality) and thus we often publish group data for residents and staff members (see Luna et al., 2021 and Luna \& Rapp, 2020). When working with our IRB members, we have found it important to establish trust based on outcomes and various measures. First, our treatment evaluations include resident satisfaction ratings of services (see Hamrick, Richling, Brogan, et al., 2021 a for an example). Second, as previously noted, we have assent procedures incorporated into our one-to-one sessions to ensure residents can opt in or out of sessions. Third, we have developed clinically driven research questions using staff- and administrator-endorsed activities and processes.

Finally, our team initially experienced difficulties in the process of disseminating the clinical research products from the juvenile justice facility. For example, some reviewers refused to read our submissions based on the claim that youth detention was immoral; others noted that our designs did not conform to traditional criteria for either single-case or group designs. Still other reviewers claimed we had selected students "on the basis of convenience" as opposed to clinical need. To address this problem, we published Brogan et al. (2018), which thoroughly explained the role of the ABA team within ABSOPP. Thereafter, we have cited this article in the method section of each article we have submitted for peer review. In addition, we hope this article has provided further clarification of our project. 


\section{Action Implications}

We recognize there are few behavior analysts working in juvenile justice. Similar to action steps outlined in Biglan (2015, 2020), in this section we provide recommendations for future and current students, practitioners, university faculty, and for citizens to increase their increase their knowledge and scope of competency with juvenile-justice involved individuals.

- For future students in behavior analysis, we recommend identifying graduate programs in which provide unique experiences (e.g., working with vocal, verbal clients, working in school or residential settings, consultative work). In addition, we encourage students to ask questions of their future faculty and supervisors about how they connect their behavior-analytic instruction and training to nontraditional settings and populations for board certified behavior analysis. As noted in Kazemi et al. (2018), students must take responsibility and ownership for their fieldwork experience. Working with underserved communities will likely present alternative career paths outside traditional, agency-based work with children on the autism spectrum.

- For students in behavior analysis and practitioners, we strongly suggest you put in antecedent and consequent strategies for contacting scholarly literature in juvenile justice research in various outlets (e.g., behavior analysis, social work, residential treatment, clinical psychology). As outlined in Briggs and Mitteer (2021), there are range of solutions to barriers when contacting the literature. In particular, we encourage students and practitioners to follow and request full-texts from researchers (some listed as authors and as cited below) via ResearchGate. ResearchGate is a free, social media platform in scholarly work is accessible to all. It may sound selfish, but researchers love disseminating their work and having informed conversations with students and practitioners. Reach out.

- For university faculty, be bold and be proactive. It is easy to prioritize your own instruction, service, and research (in fact, reinforcement contingencies surrounding tenure align with this pattern of response allocation). We know it is effortful and uncomfortable. Reach out to colleagues who research, instruct, or provide service to other populations outside of your area of expertise. Ask to sit in on a lecture or a student's thesis committee, have coffee, or even recruit their feedback on your courses or research. We need to acknowledge and appreciate other fields' perspectives and contributions from juvenile justice or elsewhere. You are likely reading this article in your office with the door closed down the hall from a colleague with meaningful experience and knowledge of a different population or setting. Make a move. It is worth it.

- Everyday citizens (which we all are) need to make collective effort to seek out opportunities and engage in self-management practices to be activists in our local communities (see Machalicek et al., 2021, for a phenomenal outline of how to do so). For our field to deliver behavior-analytic services to other populations, we need to be present and useful. Volunteer at a food pantry, 
domestic violence center, a shelter for people without housing, or residences for recovery. These are resources formally detained individuals might contact and will likely need.

\section{Conclusions and Future Directions}

The ABA team's implementation of these modules have been effective in increasing student adaptive behavior and decreasing student disruptive behavior during individualized treatment. In particular, students, teachers, therapists, staff members, and administrators report high satisfaction with our service delivery. Prior to the COVID-19 pandemic, the ABA team received increased referrals and increased acceptance of our recommendations, services, and protocols among relevant stakeholders. Although we have made substantial gains in disseminating our science and practice in a juvenile justice facility, future research should evaluate the extent to which behavior-analytic programming influences students contacting distally related consequences and environments. For example, investigators should directly assess the degree to which these skills influence (1) the frequency and quality of social interactions with authority figures (teachers, staff, probation officers), (2) students' access to privileges and therapeutic activities while in school and dormitory settings, and (3) students' overall performance in school, work, and leisure settings within the facility.

In addition to the commonly implemented interventions described above, the ABA team is currently evaluating interventions for several other behavioral concerns including impulsive decision making, social skills deficits, and academic skills deficits. In addition, we are working to increase our focus on behavioral skills that will increase the likelihood of students contacting reinforcement for appropriate behavior once they leave the facility. Recent protocols in progress include resume writing, financial literacy, specific job-related skills, and medication advocacy. Initial data analysis in these areas show promise in the development of effective treatments for these skills. We aim to evaluate the role of behavioral cusps within the facility. In particular, we are interested in examining how the acquisition of certain behavioral skills, such as appropriate reactions, might lead to access of new contingencies and environments and thereby the acquisition of new behaviors. In general, the ABA team plans to continue to address both student and staff needs on individual and group levels to improve quality of life for all those involved this juvenile justice facility. On some level, coercion as detailed by Goltz (2020) is unavoidable in detention settings. Nevertheless, we endeavor to identify coercion where it exists and minimize its influence in our behavior change processes.

Authors' Contributions All authors contributed to this submission. John Rapp had the idea for the article. The first draft of the manuscript was written by Kristen Brogan, and subsequent drafts were written by John Rapp and Odessa Luna. All authors commented on previous versions of the manuscript. All authors read, approved, and critically revised the final manuscript. 
Funding Alabama Department of Youth Services

Data Availability Not applicable

Code Availability Not applicable

\section{Declarations}

Conflicts of Interest Not applicable

\section{References}

Alabama Department of Youth Services. (n.d.). DYS diversion grant funding. Retrieved January 20, 2022, from https://dys.alabama.gov/dys_grant_funding

Anderson, A., Rapp, J. T., \& Kierce, E. (2021). Psychotropic medication prescribing in a juvenile justice facility: Evidence of a limited discontinuation process. Residential Treatment for Children \& Youth. Advance online publication. https://doi.org/10.1080/0886571X.2021.1887789

Apel, A. B., \& Diller, J. W. (2017). Prison as punishment: A behavior-analytic evaluation of incarceration. The Behavior Analyst, 40, 243-256. https://doi.org/10.1007/s40614-016-0081-6

Applebaum, K. L. (2008). Correctional mental health research: Research opportunities and barriers. Journal of Correctional Health Care, 14(4), 269-277. https://doi.org/10.1177/1078345808322607

Baum, W. M. (2017). Understanding behaviorism: Behavior, culture, and evolution ((3rd ed.). ed.). John Wiley \& Sons. https://doi.org/10.1002/9781119143673

Beavers, G. A., Iwata, B. A., \& Lerman, D. C. (2013). Thirty years of research on the functional analysis of problem behavior. Journal of Applied Behavior Analysis, 46, 1-21. https://doi.org/10.1002/jaba. 30

Betz, A. M., Fisher, W. W., Roane, H. S., Mintz, J. C., \& Owen, T. M. (2013). A component analysis of schedule thinning during functional communication training. Journal of Applied Behavior Analysis, 46(1), 219-241. https://doi.org/10.1002/jaba.23

Biggam, B. H., \& Power, K. G. (1997). Social support and psychological distress in a group of incarcerated young offenders. International Journal of Offender Therapy \& Comparative Criminology, 41, 213-230.

Biglan, A. (2015). The nurture effect: How the science of human behavior can improve our lives and our world. New Harbinger Publications.

Biglan, A. (2020). Rebooting capitalism: How we can forge a society that works for everyone. Values to Action.

Boden, L. J., Parks-Ennis, R., Allen, L., Williams, L., Williams, D., \& Dana, L. (2020). Staff and youth buy-in ideas for initial and sustainable facility-wide positive behavioral intervention and supports implementation within residential and juvenile facilities. Remedial \& Special Education, 41(2), 88-98. https://doi.org/10.1177/0741932519896078

Briggs, A. M., \& Mitteer, D. R. (2021). Updated strategies for making regular contact with the scholarly literature. Behavior Analysis in Practice, 1-12. Advance online publication. https://doi.org/10.1007/ s40617-021-00590-8

Brogan, K. M., Falligant, J. M., \& Rapp, J. T. (2017). Interdependent group contingencies decrease adolescents' disruptive behaviors during group therapy: A practitioner's demonstration. Behavior Modification, 41(3), 405-421. https://doi.org/10.1177/0145445517693812

Brogan, K. M., Richling, S. M., Rapp, J. T., Thompson, K. R., \& Burkhart, B. R. (2018). Collaborative efforts by the Auburn University applied behavior analysis program in the treatment of adolescents adjudicated for illegal sexual behavior. Behavior \& Social Issues, 27, 11-15. https://doi.org/10. 5210/bsi.v27i0.8267

Brogan, K. M., Rapp, J. T., Niedfeld, A. M., Thompson, K. R., \& Burkhart, B. R. (2020). Using arousal suppression exercises to decrease inappropriate sexual arousal in detained adolescent males: Three clinical demonstrations. Behavior Analysis in Practice, 13(2), 348-359. https://doi.org/10.1007/ s40617-020-00408-Z 
Brogan, K. M., Rapp, J. T., Edgemon, A. K., Niedfeld, A. M., Coon, J. C., Thompson, K. R., \& Burkhart, B. R. (2021). Behavioral skills training to increase appropriate reactions of adolescent males in residential treatment. Behavior Modification, 45(4), 535-559. https://doi.org/10.1177/0145445519 880837

Burkhart, B. R., Peaton, A., \& Sumrall, R. (2009). Youth services teams with universities for sex offender programs. Corrections Today, 71(3), 93-94.

Catania, A. C., \& St Peter, C. (2019). Establishing terms: A commentary on Edwards, Lotfizadeh \& Poling's "Motivating operations and stimulus control". Journal of the Experimental Analysis of Behavior, 112(1), 15-17. https://doi.org/10.1002/jeab.537

Chezan, L. C., Wolfe, K., \& Drasgow, E. (2018). A meta-analysis of functional communication training effects on problem behavior and alternative communicative responses. Focus on Autism \& Other Developmental Disabilities, 33, 195-205. https://doi.org/10.1177/1088357617741294

Chinnappan, B., Rapp, J. T., \& B. R., Burkhart. (2020). Effects of rules and feedback on classroom behavior of adolescents in a residential treatment setting. Behavior Modification, 44(5), 626-645. https://doi.org/10.1177/0145445519834637

Congress of the National Prison Association. (1870). Declaration of principles adopted and promulgated by the 1870 Congress of the National Prison Association. Retrieved December 3, 2021, from http:// www.aca.org/aca_prod_imis/docs/Exec/1870Declaration_of_Principles.pdf

Cook, J. L., Rapp, J. T., Gomes, L. A., Frazer, T. J., \& Lindblad, T. L. (2014). Effects of verbal reprimands on targeted and untargeted stereotypy. Behavioral Interventions, 29(2), 106-124. https://doi. org/10.1002/bin.1378

Cook, J. L., Rapp, J. T., \& Schulze, K. A. (2015). Differential negative reinforcement of other behavior to increase wearing of a medical bracelet. Journal of Applied Behavior Analysis, 48(4), 901-906. https://doi.org/10.1002/jaba.228

Delprato, D. J. (2002). Countercontrol in behavior analysis. The Behavior Analyst, 25(2), 191-200. https://doi.org/10.1007/BF03392057

Doughty, S. S., Anderson, C. M., Doughty, A. H., Williams, D. C., \& Saunders, K. J. (2007). Discriminative control of punished stereotyped behavior in humans. Journal of the Experimental Analysis of Behavior, 87(3), 325-336. https://doi.org/10.1901/jeab.2007.39-05

Edgemon, A. K., Rapp, J. T., Brogan, K. M., Richling, S. M., Hamrick, S. A., Peters, R. J., \& O’Rourke, S. A. (2020). Behavioral skills training to increase interview skills of adolescent males in a juvenile residential treatment facility. Journal of Applied Behavior Analysis, 53(4), 2303-2318. https://doi. org/10.1002/jaba.707

Falligant, J. M., \& Pence, S. T. (2019). Delay and probability discounting of legal outcomes. Behavior \& Social Issues, 28, 127-139. https://doi.org/10.1007/s42822-019-00011-0

First Step Act, Pub. L. No. 115-391. (2018). Retrieved January 20, 2022, from https://www.congress.gov/ bill/115th-congress/senate-bill/756

Fuller, J. L., \& Fienup, D. M. (2018). A preliminary analysis of mastery criterion level: Effects on response maintenance. Behavior Analysis in Practice, 11(1), 1-8. https://doi.org/10.1007/ s40617-017-0201-0

Galbicka, G. (1994). Shaping in the 21st century: Moving percentile schedules into applied settings. Journal of Applied Behavior Analysis, 27(4), 739-760. https://doi.org/10.1901/jaba.1994.27-739

Gendreau, P., Listwan, S. J., Kuhns, J. B., \& Exum, M. L. (2014). Making prisoners accountable: Are contingency management programs the answer? Criminal Justice \& Behavior, 41(9), 1079-1102. https://doi.org/10.1177/0093854814540288

Ghaemmaghami, M., Hanley, G. P., \& Jessel, J. (2016). Contingencies promote delay tolerance. Journal of Applied Behavior Analysis, 49(3), 548-575. https://doi.org/10.1002/jaba.333

Glenn, S. S. (1988). Contingencies and metacontingencies: Toward a synthesis of behavior analysis and cultural materialism. The Behavior Analyst, 11(2), 161-179. https://doi.org/10.1007/BF03392470

Glenn, S. S. (2004). Individual behavior, culture, and social change. The Behavior Analyst, 27(2), 133151. https://doi.org/10.1007/BF03393175

Goldiamond, I. (1976). Protection of human subjects and patients: A social contingency analysis of distinctions between research and practice, and its implications. Behaviorism, 4, 1-41 https://www. jstor.org/stable/27758852

Goldiamond, I. (1974). Toward a constructional approach to social problems: Ethical and constitutional issues raised by applied behavior analysis. Behaviorism, 2(1), 1-84.

Goltz, S. M. (2020). On power and freedom: Extending the definition of coercion. Perspectives on Behavior Science, 43, 137-156. https://doi.org/10.1007/s40614-019-00240-z 
Hamrick, S. A., Richling, S. M., Brogan, K. M., Rapp, J. T., \& Davis, W. T. (2021a). Effects of obtrusive observation and rules on classroom behavior of adolescents in a juvenile residential treatment setting. Behavior Modification, 45(5), 797-821. https://doi.org/10.1177/0145445520915676

Hamrick, S. A., Richling, S. M., Davis, W. T., Rapp, J. T., Longino, E. B., Kimber, P., Brogan, K. M., \& Law, S. (2021b). Fluency and the maintenance of skills related to sex laws for individuals adjudicated for illegal sexual behavior. Behavior Analysis in Practice, 14, 110-119. https://doi.org/10. 1007/s40617-020-00496-X

Hayes, S. C., \& Maley, R. F. (1977). Coercion: Legal and behavioral issues. Behaviorism, 5(2), 87-95 http://www.jstor.com/stable/27758895

Hockenberry, S., \& Sladkly, A. (2018). Juvenile residential facility census, 2016: Selected findings. Office of Juvenile Justice \& Delinquency Prevention. Retrieved January 20, 2022, from https://ojjdp. ojp.gov/sites/g/files/xyckuh176/files/pubs/251785.pdf

Howe, M., Clawson, E., \& Larivee, J. (2007). The 21st century: Juvenile justice work force. Corrections Today, 69, 34-39.

Iwata, B. A., Dorsey, M. F., Slifer, K. J., Bauman, K. E., \& Richman, G. S. (1994). Toward a functional analysis of self-injury. Journal of Applied Behavior Analysis, 27, 197-209. (Reprinted from Analysis \& Intervention in Developmental Disabilities, 2, 3-20, 1982) https://doi.org/10.1901/jaba.1994. 27-197

Jolivette, K., \& Nelson, C. M. (2010). Adapting positive behavioral interventions for secure juvenile justice settings: Improving facility-wide behavior. Behavior Disorders, 36(1), 28-42. https://doi.org/10. 1177/019874291003600104

Jolivette, K., Swoszowski, N. C., Parks-Ennis, R., \& Sprague, J. R. (2020). Concrete visuals for all staff within juvenile facilities. Corrections Today, 80(3), 20-26.

Kazdin, A. E. (2011). Single-case research designs: Methods for clinical and applied settings. Oxford University Press.

Kazemi, E., Rice, B., \& Adzhyan, P. (2018). Fieldwork and supervision for behavior analysts: A handbook. Springer.

Lambert, E. G. (2001). To stay or quit: A review of the literature on correctional staff turnover. American Journal of Criminal Justice, 26, 61-76.

Lambert, E. G., \& Hogan, N. (2009). The importance of job satisfaction and organizational commitment in shaping turnover intent: A test of a causal model. Criminal Justice Review, 34, 96-118.

Lane, C., Goldstein, N., Heilbrun, K., Cruise, K. R., \& Pennacchia, D. (2012). Obstacles to research in residential juvenile justice facilities: Recommendations for researchers. Behavioral Sciences \& the Law, 30(1), 49-68. https://doi.org/10.1002/bsl.1991

Laraway, S., Snycerski, S., Michael, J., \& Poling, A. (2003). Motivating operations and terms to describe them: Some further refinements. Journal of Applied Behavior Analysis, 36(3), 407-414. https://doi. org/10.1901/jaba.2003.36-407

Leaf, J. B., Cihon, J. H., Leaf, R., McEachin, J., Liu, N., Russell, N., Unumb, L, Shapiro, S., \& Khosrowshahi, D. (2021). Concerns about ABA-based intervention: An evaluation and recommendations. Journal of Autism \& Developmental Disabilities. Advance online publication. https://doi.org/ 10.1007/s10803-021-05137-y

Lechago, S. A. (2019). A comment on Edwards, Lotfizadeh, and Poling (2019) on motivating operations and stimulus control. Journal of the Experimental Analysis of Behavior, 112(1), 27-31. https://doi. org/10.1002/jeab.536

Lieberman, D. E. (2020). Exercised: Why something we never evolved to do is healthy and rewarding. Pantheon Books.

Liebling, A. (2004). Prisons and their moral performance: A study of values, quality, and prison life. Oxford University Press.

Luna, O., \& Rapp, J. T. (2020). Increasing praise delivery within dorms of a juvenile justice facility. Behavior Modification. Advance online publication. https://doi.org/10.1177/0145445520982976

Luna, O., Petri, J. M., Palmier, J., \& Rapp, J. T. (2018). Comparing accuracy of descriptive assessment methods following a brief group training. Journal of Behavioral Education, 27(4), 488-508. https:// doi.org/10.1007/s10864-018-9297-8

Luna, O., Nuhu, N. N., Palmier, J., Brestan-Knight, E., \& Rapp, J. T. (2019). Using a self-instructional package to train a group to implement reinforcement strategies. Journal of Behavioral Education, 28(3), 389-407. https://doi.org/10.1007/s10864-018-09319-0 
Luna, O., Anderson, A., \& Rapp, J. T. (2021). Resident and staff time and activity allocation in juvenile justice facility. Residential Treatment for Children \& Youth. Advance online publication. https://doi. org/10.1080/0886571X.2021.1925615

Lydon, S., Moran, L., Healy, O., Mulhern, T., \& Young, K. E. (2017). A systematic review and evaluation of inhibitory stimulus control procedures as a treatment for stereotyped behavior among individuals with autism. Developmental Neurorehabilitation, 8, 491-501. https://doi.org/10. 1080/17518423.2016.1265604

Lytle, R. (2019). Beyond panic: Variation in the legislative activity for sex offender registration and notification laws across states over time. Criminal Justice Policy Review, 30(3), 451-476. https:// doi.org/10.1177/0887403416678287

Machalicek, W., Strickland-Cohen, K., \& Drew, C. (2021). Sustaining personal activism: Behavior analysts as antiracist accomplices. Behavior Analysis Practice, Advance online publication, 1-8. https://doi.org/10.1007/s40617-021-00580-w

McDougale, C. B., Coon, J. C., Richling, S. M., O’Rourke, S., Rapp, J. T., Thompson, K. R., \& Burkhart, B. R. (2019). Group procedures for decreasing problem behavior displayed by detained adolescents. Behavior Modification, 43(5), 615-638. https://doi.org/10.1177/0145445518781314

Miguel, C. F. (2019). In defense of Jack Michael's motivation. Journal of the Experimental Analysis of Behavior, 112(1), 32-36. https://doi.org/10.1002/jeab.533

Minor, K., Wells, J. B., Angel, E., \& Matz, A. (2011). Predictors of early job turnover among juvenile correctional facility staff. Criminal Justice Review, 36, 58-75. https://doi.org/10.1177/07340 16810379253

Morris, C., Detrick, J. J., \& Peterson, S. M. (2021). Participant assent in behavior analytic research: Considerations for participants with autism and developmental disabilities. Journal of Applied Behavior Analysis, 54(4), 1300-1316. https://doi.org/10.1002/jaba.859

Neil, C. (2011). A comparison of contingent and noncontingent reinforcement for increasing passive compliance during hygiene-related tasks. [Unpublished master's thesis]. St. Cloud State University.

Niedfeld, A., Rapp, J. T., Coon, J. C., \& Cook, J. L. (2020). Using a multiple-schedule procedure to signal the availability of attention: Three demonstrations. Behavior Modification, 44(4), 496517. https://doi.org/10.1177/0145445519834640

O’Rourke, S., Richling, S., Brogan, K., McDougale, C., \& Rapp, J. T. (2021). Tolerance training in adolescent in a residential juvenile facility. Behavior Modification, 45(4), 602-618. https://doi. org/10.1177/0145445519890261

Perry, A., Koudys, J., Prichard, A., \& Ho, H. (2019). Follow-up study of youth who received EIBI as young children. Behavior Modification, 43, 181-201. https://doi.org/10.1177/0145445517 746916

Piazza, C. C., Hanley, G. P., \& Fisher, W. W. (1996). Functional analysis and treatment of cigarette pica. Journal of Applied Behavior Analysis, 29(4), 437-450. https://doi.org/10.1901/jaba.1996. 29-437

Poling, A., Lotfizadeh, A. D., \& Edwards, T. L. (2019). Motivating operations and discriminative stimuli: Distinguishable but interactive variables. Behavior Analysis in Practice, 13(2), 502-508. https://doi. org/10.1007/s40617-019-00400-2

Puzzanchera, C., \& Hockenberry, S. (2019). Trends and characteristics of youth in residential placement, 2017. Office of Juvenile Justice \& Delinquency Prevention. Retrieved January 20, 2022, from https://www.ojjdp.gov/ojstatbb/snapshots/DataSnapshot_CJRP2017.pdf

Rachlin, H. (2016). Self-control based on soft commitment. The Behavior Analyst, 39, 259-268. https:// doi.org/10.1007/s40614-016-0054-9

Rapp, J. T., Vollmer, T. R., \& Hovanetz, A. N. (2005). Evaluation and treatment of swimming pool avoidance exhibited by an adolescent girl with autism. Behavior Therapy, 36(1), 101-105. https://doi.org/ 10.1016/S0005-7894(05)80058-9

Rapp, J. T., Patel, M. R., Ghezzi, P. M., O’Flaherty, C. H., \& Titterington, C. J. (2009). Establishing stimulus control of vocal stereotypy displayed by young children with autism. Behavioral Interventions, 24(2), 85-105. https://doi.org/10.1002/bin.276

Rapp, J. T., Cook, J. L., McHugh, M., \& Mann, K. (2017). Decreasing stereotypy using NCR and DRO with functionally matched stimulation: Effects on targeted and nontargeted stereotypy. Behavior Modification, 41(1), 45-83. https://doi.org/10.1177/0145445516652370 
Reyes, J. R., Vollmer, T. R., \& Hall, A. (2011). The influence of presession factors in the assessment of deviant arousal. Journal of Applied Behavior Analysis, 44, 707-717. https://doi.org/10.1901/jaba. 2011.44-707

Ricciardi, J. N., Luiselli, J. K., \& Camare, M. (2006). Shaping approach responses as intervention for specific phobia in a child with autism. Journal of Applied Behavior Analysis, 39(4), 445-448. https:// doi.org/10.1901/jaba.2006.158-05

Richling, S. M., Williams, W. L., \& Carr, J. E. (2019). The effects of different mastery criteria on the skill maintenance of children with developmental disabilities. Journal of Applied Behavior Analysis, 52(3), 701-717. https://doi.org/10.1002/jaba.580

Rosales-Ruiz, J., \& Baer, D. M. (1997). Behavioral cusps: A developmental and pragmatic concept for behavior analysis. Journal of Applied Behavior Analysis, 30(3), 533-544. https://doi.org/10.1901/ jaba.1997.30-533

Schumacher, B. I., \& Rapp, J. T. (2011). Increasing compliance with haircuts in a child with autism. Behavioral Interventions, 26(1), 67-75. https://doi.org/10.1002/bin.321

Sidman, M. (1989). Coercion and its fallout. Authors Cooperative.

Sidman, M. (1993). Reflections on behavior analysis and coercion. Behavior \& Social Issues, 3, 75-85. https://doi.org/10.5210/bsi.v3i1.199

Smith, J. D. (2012). Single-case experimental designs: A systematic review of published research and current standards. Psychological Methods, 17, 1-41. https://doi.org/10.1037/a0029312

Sprague, J., Jolivette, K., Boden, L. J., \& Wang, E. (2020). Implementing facility-wide positive behavior interventions and supports in secure juvenile correction settings: Results of an evaluation study. Remedial \& Special Education, 41(2), 70-79. https://doi.org/10.1177/0741932519897135

Tiger, J. H., Wierzba, B. C., Fisher, W. W., \& Benitez, B. B. (2017). Developing and demonstrating stimulus control over repetitive behavior. Behavioral Interventions, 32(2), 160-174. https://doi.org/10. 1002/bin. 1472

Publisher's Note Springer Nature remains neutral with regard to jurisdictional claims in published maps and institutional affiliations. 